\title{
Pertussis vaccination and wheezing illnesses in young children: prospective cohort study
}

\author{
John Henderson, Kate North, Mancell Griffiths, Ian Harvey, Jean Golding and the Avon
}

Longitudinal Study of Pregnancy and Childhood Team

\begin{abstract}
Objectives To examine the relation between pertussis vaccination and the prevalence of wheezing illnesses in young children.

Design Prospective cohort study.

Setting Three former health districts comprising Avon Health Authority.

Subjects 9444 of 14138 children enrolled in the Avon longitudinal study of pregnancy and childhood and for whom data on wheezing symptoms, vaccination status, and 15 environmental and biological variables were available.

Main outcome measures Episodes of wheezing from birth to 6 months, 7-18 months, 19-30 months, and 31-42 months. These time periods were used to derive five categories of wheezing illness: early wheezing (not after 18 months); late onset wheezing (after 18 months); persistent wheezing (at every time period); recurrent wheezing (any combination of two or more episodes for each period); and intermittent wheezing (any combination of single episodes of reported wheezing). These categories were stratified according to parental self reported asthma or allergy.

Results Unadjusted comparisons of the defined wheezing illnesses in vaccinated and non-vaccinated children showed no significant association between pertussis vaccination and any of the wheezing outcomes regardless of stratification for parental asthma or allergy. Wheeze was more common in non-vaccinated children at 18 months, and there was a tendency for late onset wheezing to be associated with non-vaccination in children whose parents did not have asthma, but this was not significant. After adjustment for environmental and biological variables, logistic regression analyses showed no significant increased relative risk for any of the wheezing outcomes in vaccinated children: early wheezing $(0.99,95 \%$ confidence interval 0.80 to 1.23$)$, late onset wheezing $(0.85,0.69$ to 1.05$)$, persistent wheezing $(0.91,0.47$ to 1.79$)$, recurrent wheezing $(0.96,0.72$ to 1.26$)$, and intermittent wheezing (1.06, 0.81 to 1.37 ).
\end{abstract}

Conclusions No evidence was found that pertussis vaccination increases the risk of wheezing illnesses in young children. Further follow up of this population with objective measurement of allergy and bronchial responsiveness is planned to confirm these observations.

\section{Introduction}

The prevalence of asthma is increasing in various parts of the world. ${ }^{12}$ Several hypotheses have been advanced to account for this increasing prevalence including the macroenvironment (particularly atmospheric pollution) and microenvironment (such as house dust mites and household pets). A further hypothesis is that childhood vaccination programmes-in particular with pertussis-may be linked with the development of asthma. ${ }^{3}$ Odent and others reported an increased risk of asthma in a cohort of children vaccinated against pertussis that was five times greater than in a cohort of children who were not vaccinated against pertussis. ${ }^{4}$ The authors did, however, acknowledge their failure to control for potential confounding between these two populations.

Although a Swedish study, which followed up children vaccinated against pertussis until the age of 2.5 years, showed no increased risk of asthma in such children, ${ }^{5}$ other studies showed that killed Bordetella pertussis can induce a hypersensitive state in mice and rats. ${ }^{6}$ Additionally, pertussis vaccination and infection significantly increase $\operatorname{IgE}$ antibody concentrations in children. ${ }^{7}$ We investigated whether pertussis vaccination is associated with an increased incidence of recurrent wheezing in children by analysing data from the Avon longitudinal study of pregnancy and childhood, with follow up to 42 months of age.

\section{Subjects and methods}

The Avon longitudinal study of pregnancy and childhood is a prospective study of children whose mothers were enrolled during pregnancy. ${ }^{8}$ The population was defined as mothers who were resident within what is now the Avon Health Authority boundaries and who had an expected date of delivery between 1 April 1991 and 31 December 1992. An estimated $85 \%$ of all eligible pregnant women were enrolled in the study.

Information about the children's health was obtained from a structured questionnaire, which was administered to the parents at 6 months, 18 months, 30 months, and 42 months after the birth of their child. Information on whether a child had been vaccinated against pertussis was obtained from the mother. The responses were in accord with data from the Avon computerised record of child immunisations.

\section{Wheezing categories}

It is increasingly recognised that wheezing illnesses in young children represent a heterogeneous group of conditions, including asthma. For this analysis we used responses to the question, "Has your child had attacks of wheezing with whistling on the chest?" with supplementary questions regarding frequency and duration of wheezing episodes. We also used parental self reporting of a history of asthma or allergy. Children with reported wheezing episodes at defined time periods were categorised into five groups: two or more episodes of wheezing at 6 or 18 months, or both, but no wheezing at 30 or 42 months (early wheezing); two or more episodes of wheezing at 30 or 42 months, or

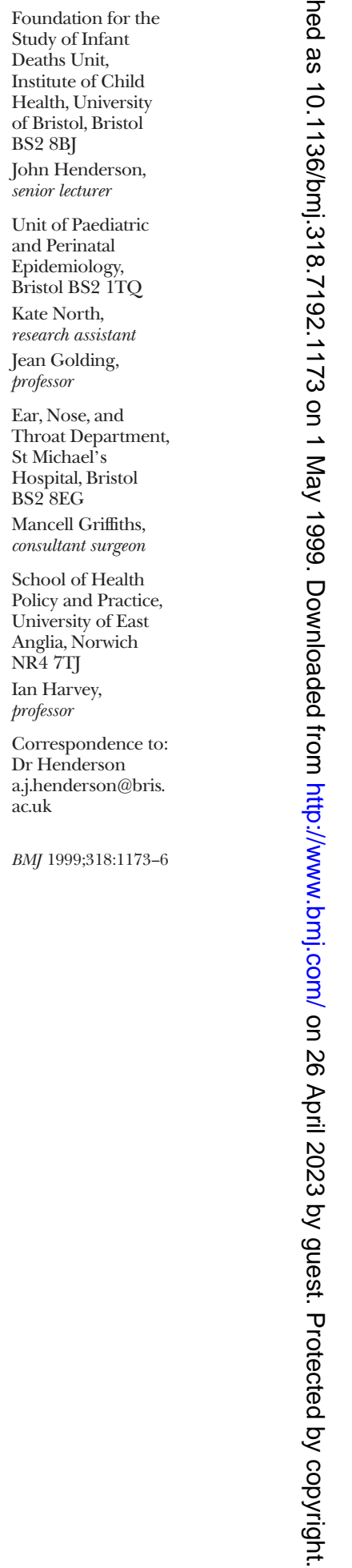


both, with no wheezing at 6 or 18 months (late onset wheezing); two or more episodes of wheezing at every time point (persistent wheezing); two or more episodes of wheezing at any combination of time periods (recurrent wheezing); and single episodes of wheezing at any time point (intermittent wheezing). These groups were further stratified according to whether either parent reported a history of asthma or allergy. Pertussis vaccination was classified as one or more vaccinations compared with no vaccinations.

\section{Statistical analyses}

We used logistic regression analysis to investigate the effects of vaccination against pertussis on the outcomes. We initially performed unadjusted logistic regression followed by multivariate analyses, which took into account: highest level of maternal education, ethnic origin of the child, number of children in the household, crowding in the home, damp or mould in the home, presence of cats in the household, financial difficulties reported by the mother, gender of the child, maternal age, duration of breast feeding, maternal smoking during pregnancy, exposure of the child to tobacco smoke, use of day care services, birth weight, and gestation. We forced vaccination into the model, and the relative risks and 95\% confidence intervals were adjusted for the other variables, which were used in a forward stepwise manner in the model.

Our sample comprised 9444 children (66.8\%) from the initial cohort of 14138 for whom data were available, with the exclusion of multiple births.

The local research ethics committees of each of the three former health districts in Avon approved the Avon longitudinal study of pregnancy and childhood, and the ethics and law committee of the Avon longitudinal study of pregnancy and childhood also approved our study.

\section{Results}

Overall, 14138 children were enrolled in the Avon longitudinal study of pregnancy and childhood. When the questionnaire was administered at 42 months, $10359(73.3 \%)$ of the children were actively participating in the study; 9444 of these children fulfilled the inclusion criteria of our study.

By 6 months of age, $88.5 \%$ of the children (8358) in the study population had been vaccinated against pertussis at least once. The prevalence of pertussis vaccination varied with several environmental and biological variables (table 1). Factors associated with increased prevalence of pertussis vaccination included increasing educational level of the mother, children born to white parents, absence of reported financial difficulties, longer duration of breast feeding, increasing maternal age, and absence of exposure to tobacco smoke in the child. There was no difference in prevalence of vaccination in low birth weight or preterm infants and no differences with gender.

The prevalence of two or more episodes of wheezing was $8.8 \%(831 / 9444)$ at 6 months after birth, $15.2 \%$ (1435) at 7-18 months, $13.1 \%$ (1237) at 19-30 months, and $10.1 \%(954)$ at $31-42$ months. We identified 924 children $(9.8 \%)$ with early wheezing, $752(8.0 \%)$ with late wheezing, $95(1.0 \%)$ with persistent wheezing, 717 $(7.6 \%)$ with recurrent wheezing, and $885(9.4 \%)$ with
Table 1 Prevalence of one or more vaccinations against pertussis in first 6 months after birth on basis of environmental and biological factors

\begin{tabular}{|c|c|c|c|}
\hline Variable & Total No & $\begin{array}{l}\text { No (\%) of } \\
\text { children }\end{array}$ & $\chi^{2}$ (P value) \\
\hline \multicolumn{4}{|c|}{ Maternal education: } \\
\hline$<0$ level & 2309 & $1851(80.2)$ & \multirow{3}{*}{$262.27(<0.0001)$} \\
\hline 0 level & 3282 & 2949 (89.9) & \\
\hline$\geqslant A$ level & 3610 & 3379 (93.6) & \\
\hline \multicolumn{4}{|l|}{ Ethnic origin: } \\
\hline White & 8667 & 7731 (89.2) & \multirow{2}{*}{$7.44(<0.001)$} \\
\hline Non-white & 357 & 302 (84.6) & \\
\hline
\end{tabular}

Number of children in household:

\begin{tabular}{|c|c|c|c|}
\hline 1 & 3961 & 3547 (89.5) & \\
\hline 2 & 3364 & $3006(89.4)$ & $8.48(<0.05)$ \\
\hline$\geqslant 3$ & 1611 & $1401(87.0)$ & \\
\hline
\end{tabular}

\section{Financial difficulties:}

\begin{tabular}{|c|c|c|c|}
\hline None & 2894 & 2634 (91.0) & \multirow{3}{*}{$21.26(<0.0001)$} \\
\hline Some & 3632 & $3218(88.6)$ & \\
\hline Many & 2532 & $2207(87.2)$ & \\
\hline \multicolumn{4}{|l|}{ Sex of child: } \\
\hline Male & 4893 & $4324(88.4)$ & \multirow{2}{*}{$0.14(0.707)$} \\
\hline Female & 4551 & $4033(88.6)$ & \\
\hline \multicolumn{4}{|c|}{ Duration of breast feeding: } \\
\hline Never & 2098 & $1756(83.7)$ & \multirow{3}{*}{$88.78(<0.0001)$} \\
\hline$\leqslant 5$ months & 4147 & $3697(89.1)$ & \\
\hline$\geqslant 6$ months & 2960 & $2727(92.1)$ & \\
\hline \multicolumn{4}{|c|}{ Exposure to tobacco smoke: } \\
\hline Yes & 3427 & $2906(84.8)$ & \multirow{2}{*}{$72.02(<0.0001)$} \\
\hline No & 6017 & $5451(90.6)$ & \\
\hline
\end{tabular}

Maternal smoking during pregnancy:

\begin{tabular}{|c|c|c|c|}
\hline None & 7746 & 6937 (89.6) & \multirow{3}{*}{$45.82(<0.0001$} \\
\hline 1-9 cigarettes/day & 723 & $621(85.9)$ & \\
\hline$\geqslant 10$ cigarettes/day & 785 & $644(82.0)$ & \\
\hline \multicolumn{4}{|l|}{ Maternal age: } \\
\hline$<25$ & 1683 & 1381 (82.1) & \multirow{3}{*}{$86.69(<0.0001)$} \\
\hline $25-29$ & 3763 & 3356 (89.2) & \\
\hline$\geqslant 30$ & 3987 & $3610(90.5)$ & \\
\hline \multicolumn{4}{|l|}{ Crowding: } \\
\hline$\leqslant 1$ person per room & 8661 & 7720 (89.1) & \multirow{2}{*}{$21.51(<0.0001)$} \\
\hline$>1$ person per room & 445 & $365(82.0)$ & \\
\hline \multicolumn{4}{|l|}{ Use of day care: } \\
\hline Yes & 1737 & 1602 (92.2) & \multirow{2}{*}{$29.20(<0.0001)$} \\
\hline No & 7707 & $6755(87.6)$ & \\
\hline \multicolumn{4}{|l|}{ Damp housing: } \\
\hline Yes & 4383 & $3969(90.6)$ & \multirow{2}{*}{$25.51(<0.0001)$} \\
\hline No & 4642 & 4048 (87.2) & \\
\hline \multicolumn{4}{|l|}{ Household cats: } \\
\hline Yes & 2598 & $2339(90.0)$ & \multirow{2}{*}{$4.03(<0.05)$} \\
\hline No & 6436 & 5691 (88.4) & \\
\hline \multicolumn{4}{|l|}{ Birth weight: } \\
\hline$<2500 \mathrm{~g}$ & 368 & $323(87.8)$ & \multirow{3}{*}{$1.74(0.419)$} \\
\hline $2500-2999 \mathrm{~g}$ & 1237 & $1082(87.5)$ & \\
\hline$\geqslant 3000 \mathrm{~g}$ & 7803 & $6920(88.7)$ & \\
\hline \multicolumn{4}{|l|}{ Gestation: } \\
\hline$<37$ weeks & 419 & $368(87.8)$ & \multirow{2}{*}{$0.19(0.664)$} \\
\hline$\geqslant 37$ weeks & 9025 & 7989 (88.5) & \\
\hline
\end{tabular}

intermittent wheezing. Table 2 shows the proportion of children with wheezing according to vaccination status at each time point. There was a tendency for increased prevalence of wheezing at 18 months in nonvaccinated children, but the relative risk for wheezing in vaccinated compared with non-vaccinated children was not significant at any time point.

The figure shows the association between pertussis vaccination and wheezing outcomes, stratified for parental asthma. Unadjusted comparisons of the 
prevalence of wheezing illnesses in vaccinated and non-vaccinated children yielded no significant associations between vaccination against pertussis and wheezing, irrespective of parental history of asthma. Late onset wheezing was more common in non-vaccinated children than vaccinated children of non-asthmatic parents, but the difference was not statistically significant. As the number of children with persistent wheezing was relatively small, we repeated the analysis using persistent wheezing with or without parental asthma as the outcome variable, and no significant association was shown. When we stratified the population according to parental self reported allergy rather than asthma similar results were produced, with no significant associations between vaccination and any of the wheezing outcomes.

Table 3 shows the results of the multiple logistic regression. Relative risks of each of the wheezing categories relative to no wheezing were calculated after adjustment for gender of the child, duration of breast feeding, maternal smoking during pregnancy, exposure of the child to tobacco smoke, mother's educational level, maternal age, number of other children, overcrowded housing, day care, birth weight, gestation, damp or mould in the home, number of cats in the household, and parental asthma.

To ensure that our observations were not confounded by the onset of wheezing before first vaccination against pertussis, we repeated the analyses for the same population but with the exclusion of all children (1801) whose wheezing pre-dated first vaccination. The results for this population were the same as those presented for children whose wheezing occurred after vaccination against pertussis, with no evidence of increased risk of wheezing illnesses in vaccinated children compared with non-vaccinated children.

\section{Discussion}

The practice of vaccination has a varied history ranging from successful eradication of smallpox to public concerns about adverse effects of vaccines. When concerns are raised about the adverse effects of vaccination, it is important to undertake a thorough investigation to either refute concerns (as in the case of the proposed links between measles vaccine and inflammatory bowel disease ${ }^{9}$ or between influenza vaccine and lung function ${ }^{10}$ ) or to describe accurately the magnitude of the adverse effect.

The tendency for pertussis antigens to stimulate IgE antibody responses has been well documented. What has been unclear is whether this effect is more pronounced after natural infection or after vaccination $^{1112}$ and whether it is clinically important. Our

Table 3 Relative risks of wheezing outcomes after adjusting for potential confounding and modifying variables

\begin{tabular}{ll} 
Wheezing category & Risk if immunised $\mathbf{( 9 5 \%} \mathbf{~ C l )}$ \\
\hline None & 1.00 (reference) \\
\hline Early & $0.99(0.80$ to 1.23$)$ \\
\hline Late onset & $0.85(0.69$ to 1.05$)$ \\
\hline Persistent & $0.91(0.47$ to 1.79$)$ \\
\hline Recurrent & $0.96(0.72$ to 1.26$)$ \\
\hline Intermittent & $1.06(0.81$ to 1.37$)$ \\
\hline
\end{tabular}

Table 2 Proportion of children who had more than two episodes of wheezing in each period according to vaccination status with pertussis

\begin{tabular}{|c|c|c|c|c|c|}
\hline \multirow{3}{*}{$\begin{array}{l}\text { Period } \\
\text { (months) }\end{array}$} & \multirow{2}{*}{\multicolumn{2}{|c|}{ No (\%) wheezing }} & \multirow[b]{3}{*}{$\chi^{2}$ (P value) } & \multicolumn{2}{|c|}{ Relative risk if vaccinated } \\
\hline & & & & \multirow{2}{*}{$\begin{array}{l}\text { Unadjusted } \\
(95 \% \mathrm{Cl})\end{array}$} & \multirow{2}{*}{$\begin{array}{l}\text { Adjusted } \\
(95 \% \mathrm{Cl})\end{array}$} \\
\hline & Vaccinated & Not vaccinated & & & \\
\hline & $n=7982$ & $\mathrm{n}=975$ & & & \\
\hline \multirow[t]{2}{*}{$0-6$} & $692(8.8)$ & $86(8.8)$ & $0.00(1.00)$ & $1.00(0.79$ to 1.26$)$ & $1.21(0.92$ to 1.60$)$ \\
\hline & $\mathrm{n}=7783$ & $\mathrm{n}=947$ & & & \\
\hline \multirow[t]{2}{*}{$7-18$} & $1157(14.9)$ & $163(17.2)$ & $3.62(0.057)$ & 0.84 (0.70 to 1.01$)$ & 0.92 (0.74 to 1.13$)$ \\
\hline & $n=8199$ & $n=1053$ & & & \\
\hline \multirow[t]{2}{*}{$19-30$} & $1054(12.9)$ & $151(14.3)$ & $1.82(0.178)$ & 0.88 (0.73 to 1.06$)$ & 0.93 (0.75 to 1.16$)$ \\
\hline & $n=7667$ & $n=911$ & & & \\
\hline $31-42$ & $770(10.0)$ & $101(11.1)$ & $0.97(0.324)$ & 0.90 (0.72 to 1.12$)$ & 0.95 (0.84 to 1.07$)$ \\
\hline
\end{tabular}

study is the first to examine the postulated links between pertussis vaccination and wheezing illness in young children after controlling for potential confounding factors. Our key finding was the absence of any difference in the adjusted prevalence of any of the three wheezing outcomes that we examined between vaccinated and non-vaccinated children.

We used wheezing illness rather than asthma as our outcome variable for several reasons. There is no clearly accepted definition of asthma on the basis of questionnaire analyses of the health of young children, and it is becoming increasingly clear that wheezing illnesses in this population are likely to represent more than one disease entity. ${ }^{13}$ We reasoned that early onset wheezing that was no longer reported by 30 months of age was likely to represent wheezing associated with respiratory infection in infancy, ${ }^{14}$ but that later onset wheezing and persistent wheezing, especially when associated with a parental history of asthma or allergy, were more likely to be representative of asthma. If vaccination against pertussis affects the prevalence of wheezing by altering immune responsiveness, particularly increasing production of $\mathrm{IgE}$ antibodies, the effect

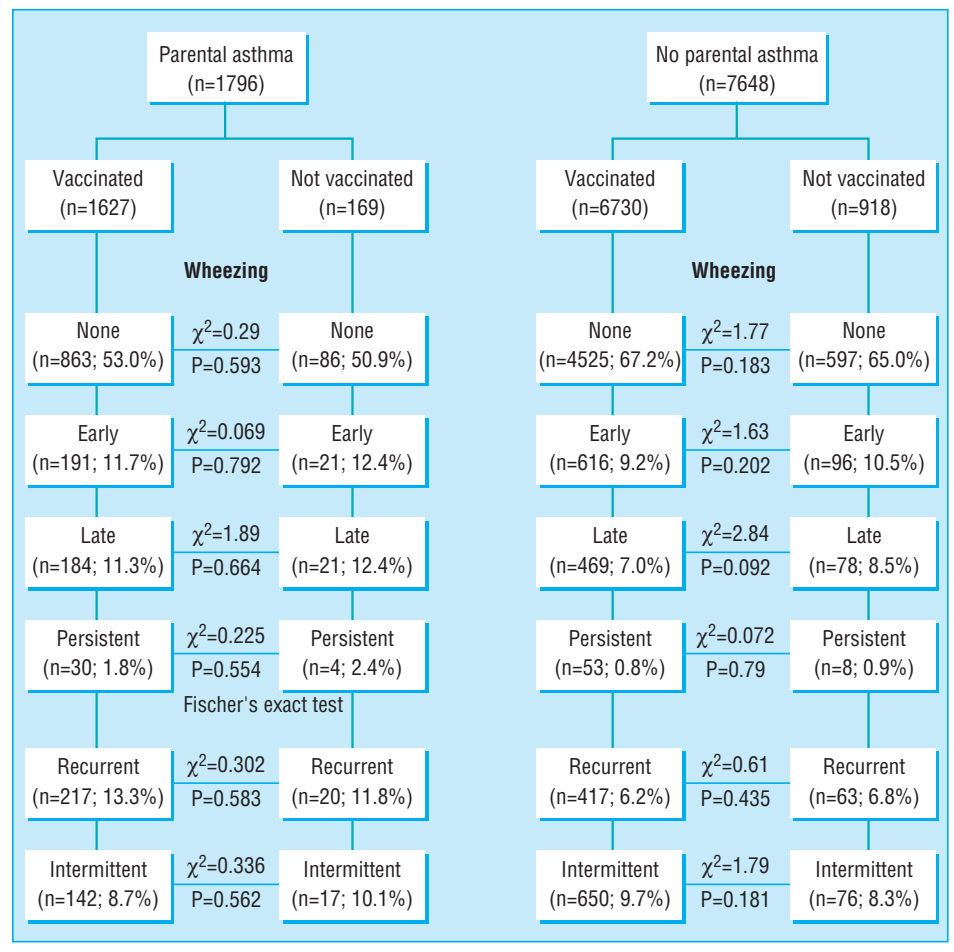

Association between pertussis vaccination and wheezing outcomes 
might be expected to be more apparent in children with asthma and allergy. We did not, however, show an increase in adjusted prevalence of wheezing with parental history of asthma or allergy in cases of either late onset wheezing or persistent wheezing who had at least one parent with a history of asthma or allergy.

Farooqi and Hopkin have recently shown an association between vaccination with whole cell pertussis vaccine and atopic disorders. ${ }^{15}$ Several important methodological differences exist between their study and ours including population selection, methods of data collection, and definitions of the principal outcome variables. Our study concentrated on one well defined respiratory symptom in young children, and we were unable to assign a definitive diagnosis of asthma on the basis of our data. However, stratification of our analysis allowed us to examine the effects of vaccination on different wheezing phenotypes, including those that are typical for wheezy bronchitis in infants and probable asthma in preschool children. We did not use diagnosis by a doctor as an independent variable in our analysis, but Farooqi and Hopkin's observations were that most children with asthma in their study were not diagnosed until after 10 years of age. Our methods are more likely to have resulted in more complete identification of cases, but we acknowledge the potential for misclassification of asthma solely on the basis of self reported data. However, those children with persistent wheezing or late onset wheezing and a parental history of asthma might reasonably be expected to be those in whom asthma is a likely explanation for their recurrent wheezing. In these two groups we found no evidence that vaccination against pertussis increased the risk of developing wheezing illness. In addition, we were able to take account of several potential confounding variables on the basis of information collected prospectively.

Further follow up of our population, with the application of objective measures of allergy and airway responsiveness, will allow us to evaluate more fully the relation between pertussis vaccination and atopic diseases. Our current results show that vaccination against pertussis is not associated with an increased prevalence of wheezing illnesses in young children, even in the presence of a family history of asthma or allergy.

We thank our funders, the midwives, and other health professionals who made the survey possible, and the parents and children who took part. Further information about the Avon longitudinal study of pregnancy and childhood can be obtained from Professor Golding or on website http:// alspac2.ich.bris.ac.uk/alspacext/.

Contributors: MG and JG had the original idea for the study and helped with the data interpretation. $\mathrm{KN}$ performed the data analyses. $\mathrm{IH}$ and $\mathrm{JH}$ helped with the data analysis and interpretation of data on wheezing. The paper was written jointly by IH, $\mathrm{JH}$, and $\mathrm{KN}$. JH and JG will act as guarantors for the paper.

Funding: The Avon longitudinal study of pregnancy and childhood is part of the European longitudinal study of pregnancy and childhood, a multicentre survey initiated by the WHO. Funding for the Avon project came from several sources including the Medical Research Council, the Wellcome Trust, the Department of Health, the Ministry of Agriculture Fisheries and Foods, and the US National Institutes of Health. JH is supported by a grant from the Foundation for the Study of Infant Deaths.

Competing interests: None declared.
Key messages

- Pertussis vaccination has been proposed as a risk factor for the development of asthma and atopy

- There was no evidence for increased wheezing illnesses in young children who were vaccinated against pertussis compared with non-vaccinated children

- Follow up studies of this population will help to further clarify the relation between early infections and vaccination and the development of atopic diseases, including asthma

- Large scale longitudinal studies beginning in pregnancy offer the opportunity to examine complex interactions between genetics and the environment in the cause of common childhood diseases

1 Burr ML, Buland BK, King S, Vaughan-Williams E. Changes in asthma prevalence: two surveys 15 years apart. Arch Dis Child 1989;64:1452-6.

2 Abramson M, Kutin J, Czarny D, Walters EH. The prevalence of asthma and respiratory symptoms among young adults: it is increasing in Australia.J Asthma 1996;33:189-96.

3 Salisbury DM, Begg NT. Immunisation against infectious disease. London: Stationery Office, 1996.

4 Odent MR, Culpin EE, Kimmel T. Pertussis vaccination and asthma: is there a link? JAMA 1994;272:592-3.

5 Nilsson L, Kjellman NIM, Storsaeter J, Gustafsson L, Olin P. Lack of association between pertussis vaccination and symptoms of asthma and allergy. JAMA 1996;275:760

6 Munoz JJ, Peacock MG. Action of pertussigen (pertussis toxin) on serum $\mathrm{IgE}$ and on Fc receptors on lymphocytes. Cell Immunol 1990;127:327-36.

7 Schuster A, Hofmann A, Reinhardt D. Does pertussis infection induce manifestation of allergy? Clin Invest 1993;71:208-13.

8 Golding J. Children of the nineties: a resource for assessing the magnitude of long-term effects of prenatal and perinatal events. Contemp Rev Obstet Gunaecol 1996;8:89-91.

9 Feeney M, Clegg A, Winwood P, Snook J. A case-control study of measles vaccination and inflammatory bowel disease. Lancet 1997;350:764-6.

10 Atmar RL, Bloom K, Keitel W, Couch RB, Greenberg SB. Effect of live attenuated cold recombinant (CR) influenza virus vaccines on pulmonary function and asthmatic adults. Vaccine 1990;8:217-24.

11 Wjst M, Dold S, Reitmeir P, Fritzsch C, von Mutius E, Thiemann H-H. Pertussis infection and allergic sensitisation. Ann Allergy 1994;73:450-4.

12 Haus M, Weinburg EG, Malherbe D. Specific IgE antibodies to Bordetella pertussis after immunisation in infancy. Lancet 1988;i:711.

13 Silverman M, Wilson N. Wheezing phenotypes in childhood. Thorax 1997;52:936-7.

14 Martinez FD, Wright AL, Taussig LM, Holberg CJ, Halonen M, Morgan WJ, et al. Asthma and wheezing in the first six years of life. $N$ Engl J Med 1995;332:133-8.

15 Farooqi IS, Hopkin JM. Early childhood infection and atopic disorder. Thorax 1999;53:927-32

(Accepted 18 February 1999)

\section{Endpieces}

\section{The hours that doctors work}

Doctors have loathsome days and hours, such as I would not wish my worst enemy. It is true that ignoramuses and coarse louts are no rarity among doctors, nor are they among writers, engineers, people in general; but those loathsome days and hours of which I speak fall to the lot of doctors only, and for that, truly, much may be forgiven them. (1891)

Letters of Anton Tchekhov, translated by Constance Garnett, London, 1920

Submitted by A L Wyman, retired physician, London 